\title{
Autocuidado e prevenção do câncer de mama: conhecimento das estudantes de graduação em saúde
}

\author{
Self-care and breast cancer prevention: knowledge of health graduation students \\ Prevención de cáncer de autocuidado y mama: conocimiento de estudiantes de \\ graduación de salud
}

Diego Augusto Lopes Oliveira ${ }^{1 *}$, Manuela Santos da Silva Diniz ${ }^{1}$, Maria Grináuria Abdon da Silva ${ }^{1}$, Elisiane Moura da Silva ${ }^{2}$, Vanessa Juvino de Sousa ${ }^{1}$, Carla Rayane Santos Dutra ${ }^{1}$, Luan José Queiroz de Lima ${ }^{1}$, Maria Eduarda Santos Silva ${ }^{1}$, Millena Rebeca Pereira de Oliveira ${ }^{1}$, Joyce Brianny da Silva ${ }^{3}$.

\section{RESUMO}

Objetivo: Descrever o conhecimento de estudantes de graduação em saúde sobre o autocuidado na prevenção do câncer de mama. Métodos: Estudo transversal e quantitativo desenvolvido em um centro universitário no Agreste pernambucano com 285 acadêmicas matriculadas em nove cursos distintos da área de ciências da saúde. A coleta dos dados se deu após anuência das participantes e utilizou questionário elaborado pelos autores, ocorrendo nos meses de agosto e setembro de 2018. Os dados coletados foram armazenados em um banco no qual se utilizou estatística descritiva e foram atribuídos conceitos mediante o agrupamento dos resultados para análise. Resultados: Observou-se que o conhecimento sobre as medidas de autocuidado e prevenção do câncer de mama, expresso pelas estudantes, foi classificado como insuficiente, ocorrendo baixa frequência das classificações regular e ótimo e não ocorrência de resultados classificados como ótimo. Conclusão: Entende-se que os aspectos relacionados a educação em saúde para empoderamento sobre questões de autocuidado são essenciais para desenvolvimento de comportamentos de busca de saúde a agravos de alta prevalência na população feminina, especialmente a de acadêmicos das ciências da saúde por lidar diretamente com questões relacionadas a saúde e adoecimento.

Palavras-chave: Neoplasias da mama, Autocuidado, Prevenção de doenças, Instituições acadêmicas, Promoção da saúde.

\begin{abstract}
Objective: To describe the knowledge of undergraduate health students about self-care in the prevention of breast cancer. Methods: Cross-sectional and quantitative study carried out at a university center in Agreste, Pernambuco, with 285 students enrolled in nine different courses in the area of health sciences. Data collection took place after the participants' consent and used a questionnaire prepared by the authors, occurring in the months of August and September 2018. The collected data were stored in a bank in which descriptive statistics were used and concepts were attributed by grouping the results. for analysis. Results: It was observed that the knowledge about the measures of self-care and prevention of breast cancer, expressed by the students, was classified as insufficient, with a low frequency of the regular and excellent classifications and nonoccurrence of results classified as excellent. Conclusion: It is understood that aspects related to health education for empowerment on issues of self-care are essential for the development of health-seeking behaviors to diseases of high prevalence in the female population, especially that of academics of health sciences for dealing directly with issues related to health and illness.
\end{abstract}

Keywords: Breast neoplasms, Self-care, Disease prevention, Schools, Health promotion.

\footnotetext{
${ }^{1}$ Centro Universitário Tabosa de Almeida (ASCES-UNITA), Caruaru - PE.

*E-mail: diegoaugusto.enf@gmail.com

${ }^{2}$ Secretaria Estadual de Saúde de Pernambuco (SES-PE), Recife - PE.

${ }^{3}$ Centro Universitário do Vale do Ipojuca (UNIFAVIP), Caruaru - PE.
} 


\section{RESUMEN}

Objetivo: Describir el conocimiento de estudiantes universitarios de salud sobre el autocuidado en la prevención del cáncer de seno. Métodos: Estudio transversal y cuantitativo realizado en un centro universitario en Agreste, Pernambuco, con 285 estudiantes matriculados en nueve cursos diferentes en el área de ciencias de la salud. La recopilación de datos se realizó después del consentimiento de los participantes y utilizó un cuestionario preparado por los autores, que se realizó en los meses de agosto y septiembre de 2018. Los datos recopilados se almacenaron en un banco en el que se utilizaron estadísticas descriptivas y se atribuyeron conceptos agrupando los resultados. para análisis. Resultados: Se observó que el conocimiento sobre las medidas de autocuidado y prevención del cáncer de mama, expresado por los estudiantes, se clasificó como insuficiente, con una baja frecuencia de las clasificaciones regulares y excelentes y la ausencia de resultados clasificados como excelentes. Conclusión: Se entiende que los aspectos relacionados con la educación en salud para el empoderamiento en temas de autocuidado son esenciales para el desarrollo de conductas de búsqueda de salud ante enfermedades de alta prevalencia en la población femenina, especialmente la de académicos en ciencias de la salud para tratar directamente con cuestiones relacionadas con la salud y la enfermedad.

Palabra-clave: Neoplasias de la mama, Autocuidado, Prevención de enfermedades, Instituciones académicas, Promoción de la salud.

\section{INTRODUÇÃO}

O Instituto Nacional de Câncer (2015) conceitua Câncer de Mama como doença causada pela multiplicação desordenada de células da mama levando a formação de um tumor. Esse tumor é o mais prevalente entre as mulheres alcançando 2,1 milhões de casos novos em todo o mundo e equivalendo a $11,6 \%$ de todos os canceres estimados. As maiores taxas de incidência esperadas ocorrem na Austrália e Nova Zelândia, nos países do Norte da Europa e na Europa Ocidental. Estima-se que no Brasil haverá 66.280 casos novos de câncer de mama, para cada ano do triênio 2020-2022. Esse valor corresponde a risco estimado de 61,61 casos novos a cada 100 mil habitantes, sendo essa patologia a primeira causa de morte, por câncer, entre as mulheres (INCA, 2019).

Estes dados refletem que ainda há muito a ser feito pelos profissionais de saúde no que tange a promoção da saúde e a prevenção deste agravo na população. Desta forma ressalta-se a importância da criação de estratégias e programas que visem à redução do número de casos do agravo citado, despertando nas mulheres o interesse para o autocuidado, prevenção primaria através da mudança de hábitos de vida e para a realização dos exames de rastreamento do câncer de mama por meio da efetivação das atividades educativas (MENDES LC, et al., 2017).

Baseado nesses pressupostos o Ministério da Saúde estabelece frentes no desenvolvimento de ações que possibilitem medidas de promoção da saúde e prevenção da doença nos cenários de atenção através do desenvolvimento de políticas de saúde específicas para o câncer de mama (MIGOWSKI A, et al., 2018).

As diretrizes para rastreamento e detecção precoce deste tipo de câncer no Brasil privilegiam além de medidas como estas a realização de ações educativas para fortalecimento da iniciativa e capacidade de autocuidado que levem as mulheres a desenvolver comportamentos positivos na prevenção da doença e promoção de hábitos de vida que privilegiem o bem-estar e manutenção de sua saúde.

A linha de cuidado estabelecida para mulher, no tocante ao rastreamento e detecção precoce, compreende medidas relacionadas ao desenvolvimento do Autoexame das Mamas (AEM), de iniciativa pessoal e, fomentando a busca dos serviços de saúde para avaliação especializada através do Exame Clínico das Mamas (ECM), sendo esta uma avaliação realizada por profissional habilitado e que orienta a realização de exames complementares de acordo com a especificidade de cada caso avaliado. O desenvolvimento destas iniciativas é de baixo custo, fácil operacionalização e possibilitam a investigação para o diagnóstico da doença de forma mais precoce, garantindo melhor prognóstico a mulher acometida (INCA, 2017). 
Para Santos JS, et al. (2019) observa-se fragilidade no conhecimento das mulheres sobre as medidas preventivas do câncer de mama, tal fato tem uma grande pertinência pois um bom conhecimento tem repercussões positivas na prevenção, na mudança do estilo de vida e até mesmo na detecção precoce da doença. Entende-se, enquanto consagrado na literatura, que medidas de propagação do conhecimento através da educação e saúde e suas tecnologias e das iniciativas de ações de prevenção primária levam a mulher a empoderamento acerca da sua situação de saúde e estilo de vida, diminuindo os fatores de risco que são associados ao câncer (KRIST AH, et al., 2017).

Diante do pressuposto, este estudo tem como objetivo descrever o conhecimento das estudantes de graduação em saúde sobre o autocuidado para prevenção do câncer de mama, visto que, nas ciências da saúde os profissionais comumente apresentam domínio técnico e científico para cuidado de outro ser humano. Os aspectos relacionados à rotina de estudo/trabalho, vida social/pessoal e condições psicoafetivas afastam essas mulheres do desenvolvimento de medidas preventivas causando consequências à manutenção de sua saúde. É essencial que o profissional cuide de si contribuindo positivamente não só para fortalecimento do seu autocuidado, mas também potencializando sua aptidão e empoderamento frente ao processo de trabalho como educador em saúde.

\section{MÉTODOS}

Trata-se de um estudo descritivo, transversal com abordagem quantitativa desenvolvido em um centro universitário da cidade de Caruaru-PE. Nesta instituição é desenvolvido ensino em cursos de graduação na área das ciências da saúde, sendo ofertadas vagas para formação em Biomedicina, Educação Física (Bacharelado e Licenciatura), Enfermagem, Farmácia, Fisioterapia, Nutrição, Odontologia e Saúde Coletiva.

A amostra incluiu estudantes do sexo feminino matriculadas nos referidos cursos de graduação, maiores de 18 anos e que estivessem em curso regular do segundo semestre letivo do ano de 2018. Considerou-se como critério de exclusão as estudantes que passaram por tratamento para câncer de mama e as que possuíam o curso de graduação atual como segunda formação na área de saúde.

Para definição do tamanho da amostra foi utilizado cálculo amostral considerando nível de confiança de $95 \%$, proporção esperada de 0,50 , margem de erro de 0,05 e população total de 1348 estudantes do sexo feminino, compondo uma amostra total de 299 estudantes.

A coleta dos dados aconteceu nos meses de agosto e setembro de 2018 onde foram captadas 299 estudantes, no entanto depois de aplicados os critérios de elegibilidade, totalizaram-se 285 como elegíveis para participação na pesquisa.

A partir desse dado foi realizada amostragem estratificada de estudantes do sexo feminino, separadamente por cada curso de graduação, para estabelecimento do quantitativo de observações por curso, este respeitando a prevalência inicial de estudantes em cada graduação, isoladamente.

A seleção das estudantes foi proporcional ao número de estudantes de cada sala, sendo as participantes selecionadas a partir de amostragem aleatória por seriado simples, utilizando a frequência aplicada em sala de aula para sorteio de um número e partir deste foi sequenciado em três.

A coleta foi instrumentada por meio de questionário elaborado pelos autores estruturado em duas partes: a primeira composta por questões tratando de informações pessoais (idade, curso, profissão, escolaridade) fatores de riscos percebidos para a doença, fatores de risco relacionados ao ciclo reprodutivo da mulher (menarca, nuliparidade, números de consultas médicas ou de enfermagem) e atitudes relacionadas ao autocuidado e à detecção precoce (autoexame das mamas, exame clínico das mamas por profissional habilitado e exame mamográfico).

Antes de sua efetiva aplicação junto a população alvo o instrumento passou por teste piloto e foi aplicado entre 20 estudantes do curso de Direito da mesma instituição no mês de maio de 2018. A aplicação otimizou reformulações de conteúdo e escrita para melhor compreensão e alcance dos objetivos propostos no estudo. 
Os dados coletados foram organizados em um banco no programa Microsoft Excel for Windows (versão 2010) e calculadas as frequências absoluta e relativa. Estes foram analisados de acordo com a frequência atingida nas respostas corretas e incorretas frente às recomendações para rastreamento e diagnóstico precoce do câncer de mama preconizadas pelo Ministério da Saúde do Brasil e Instituto Nacional do Câncer (INCA, 2017).

Para critério de análise foi atribuído pontuação por questão (1,0 ponto) perfazendo um total de 10 questionamento. Após aplicação da pontuação dos questionários estes foram classificados por conceitos obedecendo o seguinte critério de agrupamento: questionários com pontuação inferior a 7 pontos: Insuficiente; pontuação de 7 pontos: Regular; pontuação de 8 pontos: Bom e pontuação entre 9 e 10 pontos: Ótimo.

Pela sua característica geral o estudo exigiu observância quanto ao cumprimento das normativas expressas na Resolução no 466/2012 do Conselho Nacional de Saúde. Todas as participantes assinaram Termo de Consentimento Livre e Esclarecido (TCLE), sendo informadas dos riscos e benefícios gerados pela pesquisa, bem como das formas de minimizá-los e seus direitos enquanto participante. $O$ estudo foi aprovado pelo Comitê de Ética em Pesquisa (CEP) do Centro Universitário Tabosa de Almeida (ASCES/UNITA) sob o CAAE no: 86024418.6.0000.5203 e Parecer no 2.674.228.

\section{RESULTADOS}

Evidenciou-se que a faixa etária de maior concentração das participantes foi entre 20 a 29 anos, denotando $174(61 \%)$ mulheres. A média das idades foi de 21 anos, sendo a idade mínima de 17 anos, a máxima de 41 anos, identificando uma mediana de 21 anos. Com referência ao estado civil, 249 (87\%) declarou-se solteira. Em relação à cor ou raça, foram identificadas em maior frequência para raça branca $138(48,4 \%)$, seguida da raça parda $127(44,6 \%)$. Quanto ao turno de estudo a maioria frequentava a IES em turno noturno 148 (52\%). Identificou-se que $172(60,4 \%)$ apresentou menarca entre 10-12 anos. Não tinham filhos $260(91 \%)$ e 262 (92\%) relataram não ter desenvolvido amamentação. Observou-se que $212(74 \%)$ afirmaram não possuir risco para câncer de mama (Tabela 1).

Tabela 1 - Perfil sociodemográfico das estudantes dos cursos de saúde, n=285.

\begin{tabular}{cccc}
\hline Variável & & $\mathbf{n}$ & $\%$ \\
\hline \multirow{3}{*}{ Idade } & <20 Anos & 93 & 32,6 \\
& 20 A 29 Anos & 174 & 61 \\
& 30 A 39 Anos & 16 & 5,6 \\
\multirow{2}{*}{ Estado Civil } & $>40$ Anos & 02 & 0,7 \\
& Solteira & 249 & 87 \\
& Casada & 31 & 11 \\
& Divorciada & 05 & 2 \\
\hline \multirow{2}{*}{ Raça } & Branca & 138 & 48,4 \\
& Parda & 127 & 44,6 \\
& Afrodescendente & 09 & 3,1 \\
& Amarela & 09 & 3,1 \\
& Indígena & 02 & 0,7 \\
\hline \multirow{3}{*}{ Turno de Estudo } & Diurno & 21 & 7,4 \\
& Vespertino & 18 & 6,3 \\
& Diurno/Vespertino & 98 & 34,3 \\
& Noturno & 148 & 52 \\
\hline \multirow{2}{*}{ Menarca } & $<10$ Anos & 07 & 2,4 \\
& 10 A 12 Anos & 172 & 60,4 \\
& $>12$ Anos & 106 & 37,2 \\
\hline \multirow{2}{*}{ Filhos } & Sim & 25 & 9 \\
& Não & 260 & 91 \\
\hline \multirow{2}{*}{ Amamentação } & Sim & 23 & 8 \\
& Não & 262 & 92 \\
\hline Risco para Câncer de & Sim & 73 & 26 \\
Mama & Não & 212 & 74 \\
\hline
\end{tabular}

Fonte: Oliveira DAL, et al., 2020. 
A seguir descrevem-se os resultados relacionados ao desempenho das estudantes dos cursos de saúde frente ao autocuidado na prevenção do câncer de mama, focando a importância de se ter conhecimento dos fatores de risco assim como os métodos de rastreamento e detecção precoce da doença. Após reunir e pontuar todas as respostas das estudantes, identificando acertos e erros, classificou-se o resultado final obtido em categorias considerando a pontuação obtida no total de acerto das questões.

O apanhado do desempenho geral evidenciou que as estudantes dos cursos Educação Física Bacharelado, Educação Física Licenciatura, Nutrição e Saúde Coletiva apresentaram desempenho insuficiente ( $<7$ pontos no total). Houve desempenho classificado em mais de uma categoria (Insuficiente, Regular e Bom) nos cursos de Biomedicina, Enfermagem, Farmácia, Fisioterapia e Odontologia. Nenhuma das estudantes participantes da pesquisa apresentou resultado classificado como ótimo (entre 9 e 10 pontos) na avaliação (Tabela 2).

Tabela 2 - Distribuição percentual do desempenho atingido pelas estudantes do curso de saúde na avaliação, $n=285$.

\begin{tabular}{cccccc}
\hline Curso & $\mathbf{n}$ & Insuficiente & Regular & Bom & Ótimo \\
\hline Biomedicina & 27 & $96,3 \%$ & - & $3,7 \%$ & - \\
Ed. Física & 18 & $100 \%$ & - & - & - \\
Bacharelado & & & & & \\
Ed. Física & 08 & $100 \%$ & - & - & - \\
Licenciatura & 50 & $82 \%$ & $14 \%$ & $4 \%$ & - \\
Enfermagem & 50 & $93,1 \%$ & $6,9 \%$ & - & - \\
Farmácia & 58 & $97,9 \%$ & $2,1 \%$ & - & - \\
Fisioterapia & 47 & $100 \%$ & - & - & - \\
$\quad$ Nutrição & 11 & $95,2 \%$ & $4,8 \%$ & - & - \\
Odontologia & 63 & $100 \%$ & - & - & - \\
$\quad$ Saúde & 03 & - & - & - & - \\
Coletiva & & & &
\end{tabular}

Fonte: Oliveira DAL, et al., 2020.

\section{DISCUSSÃO}

Em seu conjunto de dados a aplicação do instrumento evidenciou um perfil de estudantes com características consideradas de risco para o desenvolvimento do câncer mamário especialmente quando relacionados a raça (branca), menarca precoce e nuliparidade. A faixa etária prevalente não se configura como de risco, conforme a elucidação das estimativas nacionais para o acometimento dessa doença (INCA, 2019). Em estudo aplicado entre 50 estudantes de ambos os sexos dos cursos de Psicologia, Fisioterapia e Enfermagem na avaliação do conhecimento acerca dos fatores de risco para o câncer de mama observou-se perfil semelhante ao da amostra deste estudo, sendo a população do referido estudo composta, em sua maioria, por estudantes do sexo feminino, de cor branca e nulíparas (PIRHARDT CR e MERCÊS NNA, 2009).

No que se relaciona a raça referida pela participante no momento da pesquisa a maioria se denominou branca, tendo esse quantitativo se sobressaído em relação as mulheres pardas. A prevalência e susceptibilidade de surgimento deste tipo de câncer são maiores nas mulheres de pele branca, porém o diagnóstico tardio é realizado mais frequentemente na população afrodescendente (NUNES BAP, et al., 2012) (GUIMARÃES AGC e ANJOS ACY, 2012). Além disso outro dado relevante é que em estudo de coorte realizado em 239 hospitais brasileiros constataram-se atrasos no diagnóstico e tratamento desta neoplasia em mulheres com a cor diferente da branca, evidenciando influência das características étnicas manejo do diagnóstico precoce (MEDEIROS GC, et al.,2015).

A idade é um fator de risco devido ao envelhecimento, bem como ao tempo de exposição aos carcinógenos. Quanto mais se avança na idade cronológica, o risco de exposição principalmente ambiental aumenta e, como o câncer de mama é multicausal, fatores associados aumentam a possibilidade do desenvolvimento da doença (ARRUDA LA, et. al., 2015). Em relação ao turno de estudo, o noturno 
prevaleceu, sendo uma opção das estudantes para assim durante o dia exercerem suas atividades como o trabalho, um fator que influencia diretamente na disponibilidade para desenvolver o seu autocuidado.

Em estudo multicêntrico realizado em dois hospitais públicos federais do Rio Grande do Sul, Brasil, com 175 mulheres investigando hábitos alimentares e perfil nutricional e sua interferência no câncer de mama observou-se a prevalência de menarca aos 13 anos de idade. Na amostra desta pesquisa a menarca evidenciou intervalo de ocorrência em idade inferior aos 12 anos de idade, sendo considerada fator de risco preponderante para o diagnóstico desta tumoração (NUNES BAP, et al., 2012).

A menarca precoce é considerada fator de risco devido à exposição ao estrogênio. As mulheres que têm menarca precoce e estabelecem rapidamente um ciclo regular têm risco, uma vez que os níveis de estrogênio são maiores durante a fase lútea normal, e o índice de exposição acumulativa ao estrogênio é maior (DERENZO N, et. al., 2017).

A consolidação desse perfil aliado aos hábitos de vida desenvolvidos por estas estudantes, não investigados neste estudo, podem influenciar significativamente a ocorrência desta neoplasia entre essas mulheres. Há de se considerar que outros fatores na característica clínica foram evidenciados e compõem um perfil com risco para o desenvolvimento desta neoplasia especialmente características como mulheres que nunca engravidaram ou nunca tiveram filhos, uso de anticoncepcionais orais, consumo de bebidas alcoólicas, sedentarismo e consumo de dieta hipercalórica.

Considerando os conceitos obtidos na avaliação global do conhecimento sobre os métodos de autocuidado e prevenção para o câncer de mama foi evidenciado nível de conhecimento insuficiente como mais frequente entre as participantes do estudo, especialmente quando relacionado à compreensão dos fatores de risco bem como os métodos de rastreamento e detecção precoce da doença.

Os resultados demonstraram que as estudantes dos cursos Educação Física Bacharelado, Educação física Licenciatura, Nutrição e Saúde Coletiva apresentaram desempenho insuficiente, levando a pensar que essas estudantes não vivenciam este conteúdo durante o ensino superior ou não recebem orientação para essa prática em algum momento da graduação, haja vista que esses cursos não apresentam um perfil voltado para o desenvolvimento da atividade de prevenção e autocuidado para o câncer de mama como rotineira em sua prática profissional.

Evidenciou-se, ainda, que as estudantes dos cursos de Biomedicina, Enfermagem, Farmácia, Fisioterapia e Odontologia apresentaram desempenho em mais de uma categoria de classificação. $O$ curso que apresentou o melhor desempenho de conceitos foi o de Enfermagem, pois possui em sua constituição curricular unidade temática que aborda o programa de atenção à saúde da mulher e enfatiza as práticas de rastreamento e detecção precoce desta neoplasia. Outro fator importante para o desempenho diferenciado em relação aos demais cursos é a existência de projeto de extensão que aborda o tema e os métodos preventivos, onde a maior parcela de estudantes é do curso de enfermagem.

Estudo realizado no Iraque com mulheres da população acadêmica (docentes, discentes e corpo administrativo) de duas universidades, através da aplicação de instrumento da avaliação de conhecimento, constatou altos percentuais de baixa pontuação de conhecimento e baixa ocorrência de conhecimento classificado como "Bom" e acima, se assemelhando ao evidenciado na amostra deste estudo. Constatou-se ainda que as participantes acreditavam que a melhor maneira de controlar o câncer de mama é a detecção precoce e de outras possíveis medidas preventivas, sendo referido a falta de conhecimento como motivo mais comum para não as desenvolver (ALWAN NAS, et al., 2012)

Um estudo realizado com o total de 296 entrevistados em uma universidade do Rio Grande do Norte (RN), os cursos de Enfermagem, Fisioterapia e Nutrição detectou que os acadêmicos tinham um bom conhecimento sobre a prevenção do câncer de colo do útero e de mama, diferentemente deste estudo em que o curso de nutrição, houve um conhecimento insuficiente. O conhecimento acerca do câncer de útero e mama, contendo os sinais e sintomas, métodos de prevenção, diagnóstico e tratamentos destacam-se como importantes na prática profissional e para o autocuidado (ROCHA JPJ, et al., 2018). Visto isso, percebe-se a necessidade de melhorar abordagem desta temática nos cursos referidos para que as estudantes sejam capazes de se autocuidar e de desenvolver competências de educadores em saúde para o exercício da profissão. 
O desconhecimento sobre estes fatores associados ao autocuidado se configura como uma grande barreira para ação dos profissionais no estabelecimento do diagnóstico precoce, inserção da mulher em tratamento e em bom prognóstico. O Ministério da Saúde e o INCA promovem continuamente elaboração e atualização de consenso para práticas de rastreamento e detecção precoce do câncer de mama de forma a estimular os serviços de saúde, especialmente, os relacionados à atenção básica a promoverem medidas de orientação e fortalecimento do conhecimento das mulheres quanto aos fatores de risco e fortalecimentos dos ideais de promoção à saúde e prevenção deste agravo (MIGOWSKI A, 2016).

Em estudo realizado entre mulheres, em faixa predominantemente similar a este estudo, sobre métodos de prevenção para o câncer de mama evidenciou-se que quase metade das entrevistadas não conhece nenhum fator de risco para a doença, e entre as que possuem conhecimento há relatos de apenas um fator e uma parcela mínima (menos de $5 \%$ ) referem dois ou mais fatores de risco para a doença.

Tais resultados quando analisados junto às informações das boas práticas divulgadas pelo ministério da saúde, através dos programas de atenção básica, sugerem que os fatores de risco são pouco abordados nas ações de educação em saúde junto a esta população (BATISTON AP, et. al., 2011).

A educação ofertada a mulher, tendo em vista a prevenção e detecção precoce do tumor, não deve se constituir na simples transmissão de informação, mas deve ter embasamento científico não deixando de lado o contexto sociocultural, valores, crenças e conhecimento popular (RODRIGUES AB, OLIVEIRA PP, 2016).

O desconhecimento dos fatores de risco na amostra remete a possíveis prejuízos a capacidade de autocuidado, pois a informação potencializa a adoção de medidas autônomas para o cuidar de si mesmo. É imprescindível que o profissional de saúde esteja atento para perceber os limites que impedem as mulheres de dimensionar suas dificuldades em relação ao câncer de mama, bem como identificar estratégias que possibilitem às mulheres com maior risco, a adoção de práticas de autocuidado, como a adoção de hábitos de vida saudável, realização regular do autoexame das mamas, de exame clínico e da mamografia (OLIVEIRA DAL, 2019) (MENDES LC, et. al., 2017).

Este estudo encontrou como limitação a baixa disponibilidade de fontes científicas atualizadas voltadas a prevenção entre estudantes de graduação e a relação com o autocuidado. As fontes disponíveis normalmente abordam a mulher com câncer de mama em cenários de reabilitação da doença, em tratamento ou nos serviços de atenção em média e alta complexidade.

Desta forma, enfatiza-se a necessidade de desenvolver estudos que promovam a elucidação do conhecimento sobre estas práticas de autocuidado na prevenção deste câncer entre todas as mulheres envolvidas na rotina acadêmica, em especial as docentes, de forma a estimular a disseminação da informação e meios de potencialização do autocuidado no interior das universidades de forma a expandir essas ações para a comunidade no formato de projetos, ações e iniciativas que perpassem períodos anuais e tornem-se rotina de forma a contribuir na redução dos malefícios ocasionados pelo diagnóstico tardio e o sofrimento associado aos tratamentos desta doença.

\section{CONCLUSÃO}

A prevenção do câncer de mama ainda é primária e a prática do autocuidado deve ser estimulada, por ser uma das maneiras mais acessíveis no combate e controle deste tipo de câncer. Salienta-se a importância das medidas preventivas para o controle do câncer de mama baseadas em atitudes de autocuidado, destacandose a necessidade das acadêmicas vivenciarem esta temática ainda na academia, uma vez que a instituição enquanto agente formativo deve garantir a entrega, aos serviços de saúde, de profissionais com conhecimentos adequados para desenvolvimento de uma prática educativa e assistencial voltada a benefícios a saúde da população feminina.

\section{REFERÊNCIAS}

1. ALWAN NAS, et al. Knowledge, attitude and practice regarding breast cancer and breast self-examination among a sample of the educated population in Iraq. East Mediterr Health J. 2012; 18(4):337-45. 
2. ARRUDA LA, et al. Prevenção do câncer de mama em mulheres atendidas em Unidade Básica de Saúde. Rev. Rene, 2015; 16(2):143-9.

3. BATISTON AP, et al. Conhecimento e prática sobre os fatores de risco para o câncer de mama entre mulheres de 40 a 69 anos. Rev. Bras. Saúde Matern. Infant., 2011; 11(2).

4. DERENZO N, et al. Conhecimento de mulheres sobre fatores relacionados ao câncer de mama. Rev. Enferm. UFSM, 2017; 7(3):436-447.

5. GUIMARÃES AGC, ANJOS ACY. Caracterização sociodemográfica e avaliação da qualidade de vida em mulheres com câncer de mama em tratamento quimioterápico adjuvante. Rev Bras Cancerol. 2012; 58(4).

6. INSTITUTO NACIONAL DE CÂNCER JOSÉ ALENCAR GOMES DA SILVA. Estimativa 2020: incidência de câncer no Brasil / Instituto Nacional de Câncer José Alencar Gomes da Silva - Rio de Janeiro: INCA, 2019.

7. INSTITUTO NACIONAL DE CÂNCER JOSÉ ALENCAR GOMES DA SILVA. Diretrizes para a detecção precoce do câncer de mama no Brasil/ Instituto Nacional de Câncer José Alencar Gomes da Silva - Rio de Janeiro: INCA, 2015.

8. INSTITUTO NACIONAL DE CÂNCER JOSÉ ALENCAR GOMES DA SILVA. Diretrizes para a detecção precoce do câncer de mama no Brasil - Sumário Executivo. Instituto Nacional de Câncer José Alencar Gomes da Silva - Rio de Janeiro: INCA, 2017.

9. KRIST AH, et al. Harnessing Information Technology to Inform Patients Facing Routine Decisions: Cancer Screening as a Test Case. The Annals of Family Medicine, 2017;15 (3) 217-224.

10. MEDEIROS GC, et al. Análise dos determinantes que influenciam o tempo para o início do tratamento de mulheres com câncer de mama no Brasil. Cad. Saúde Pública, 2015; 31(6):1269-1282.

11. MENDES L, et al. Conhecimento e prática da autopalpação das mamas entre estudantes de escolas públicas do período noturno. Rev Enferm UERJ, 2017; 25: e13746.

12. MENDES LC, et al. Atividades educativas estimulando o autocuidado e prevenção do câncer feminino. Rev. Enferm Atenção a Saúde, 2017. 6(1):140-147.

13. MIGOWSKI A, et al. Diretrizes para detecção precoce do câncer de mama no Brasil. II- Novas recomendações nacionais, principais evidências e controvérsias. Cad. Saúde Pública, 2018; 34(6): e00074817.

14. MIGOWSKI A. A interpretação das novas diretrizes para detecção precoce do câncer de mama no Brasil. Cad. Saúde Pública, 2016; 32(8).

15. NUNES BAP, et al. Perfil epidemiológico dos pacientes diagnosticados com câncer de mama em Campos dos Goytacazes (RJ), Brasil. Rev Bras Mastologia, 2012; 22(4).

16. OLIVEIRA DAL. Educação em saúde no autocuidado contra o câncer de mama. Rev. EnfermAtual in derme, 2019; 87(25).

17. PIRHARDT CR, Mercês NNA. Fatores de risco para câncer de mama: nível de conhecimento dos acadêmicos de uma universidade. Rev. Enferm. UERJ, 2009; 17(1).

18. ROCHA JPJ, et al. Conhecimento de acadêmicos sobre a prevenção do câncer de colo do útero e de mama. Rev. Enferm. UFSM, 2018; 8(3).

19. RODRIGUES AB, OLIVEIRA PP. Oncologia para enfermagem. Rio de Janeiro: Editora Manole; 2016.

20. SANTOS JS, et al. O conhecimento das mulheres sobre a prevenção do câncer de mama. Brazilian Journal of surgery and clinical research, 2019; 29(3): 15-21. 